\title{
SUSY and BSM physics prospects at the high-luminosity LHC
}

\section{Barbara Clerbaux* ${ }^{\dagger}$}

IIHE-ULB, Université Libre de Bruxelles, ULB

Brussels, Belgium

E-mail: barbara.clerbauxecern.ch

\begin{abstract}
Prospects on searches for new physics at the high-luminosity LHC are presented for the ATLAS and CMS experiments. Two integrated luminosity scenarios are assumed : $300 \mathrm{fb}^{-1}$, corresponding to the end of the LHC run 3 , and $3000 \mathrm{fb}^{-1}$, the integrated luminosity expected to be collected during the LHC phase 2. Results for strongly produced and weakly produced supersymmetric particles are first detailed. A selection of results on searches for new physics beyond the standard model is then given, in particular the search for heavy resonances in various decay channels and the search for dark matter candidates.
\end{abstract}

Fourth Annual Large Hadron Collider Physics

13-18 June 2016

Lund, Sweden

\footnotetext{
*Speaker.

${ }^{\dagger}$ On behalf of the ATLAS and CMS Collaborations
} 


\section{Introduction}

While physicists from the ATLAS and CMS experiments [1,2] are involved in the data analysis of the LHC run 1 (data taken in years 2010-2012) and of the new high energy run (LHC run 2) that started in 2015 , they are in addition actively preparing the long term future of the LHC, studying the physics potential of its high luminosity phase, the so called HL-LHC (also known as the LHC phase 2 upgrade). Dedicated web pages have been developed by ATLAS and CMS and are given in Refs. [3,4]. The HL-LHC is expected to start taking data in 2026 for about 10 years, the nominal instantaneous luminosity is expected to be $5 \times 10^{34} \mathrm{~cm}^{-2} \mathrm{~s}^{-1}$, with an average number of proton-proton (pp) interactions per bunch crossing (pileup) $\langle\mu\rangle$ of about 140 , leading to an integrated luminosity of $250 \mathrm{fb}^{-1}$ per year. Recently a new goal was proposed targeting an ultimate luminosity of $7.5 \times 10^{34} \mathrm{~cm}^{-2} \mathrm{~s}^{-1}$, with $\langle\mu\rangle=200$ and more than $300 \mathrm{fb}^{-1}$ accumulated per year. To sustain this very harsh machine conditions, the ATLAS and CMS detectors have to be significantly upgraded, with some part of it, as the tracker detector, entirely replaced. The description and performance of the upgraded detectors are available in the Technical Proposal and scoping documents reported in Refs. [5-7]. In these proceedings the physics potential for the search of new physics at the end of run 3 (corresponding to $300 \mathrm{fb}^{-1}$ of data) and at the end of the HL-LHC phase 2 (3000 $\mathrm{fb}^{-1}$ of data) is studied at a center of mass energy $\sqrt{s}=14 \mathrm{TeV}$. For the event simulation of standard model (SM) backgrounds and signal processes, several full upgraded detector simulations exist, but most results presented here are based on fast simulation programs. In that case, the detector response is a parametrisation of the upgraded detector performance extracted from Monte-Carlo (MC) samples using the full detector simulation in a high pileup condition, mainly $\langle\mu\rangle=140$ or 200 . The parametrisations describe the reconstruction efficiencies and the momentum resolutions for electrons, muons, $b$-jets and for the missing transverse energy (MET) variable, as well as misidentification rates.

\section{Prospects on SUSY searches}

Prospects of SUSY searches in the context of the HL-LHC have been investigated in detail by both ATLAS and CMS collaborations and results are reported in Refs. [7-10] and [5, 6, 11, 12], respectively. Only a part of these results is presented in these proceedings: first searches for SUSY topologies from strong production are reported, followed by the ones from electroweak production. The results are generally interpreted in the context of simplified models, in which the SUSY particle under consideration is assumed to decay to a SM particle and the neutralino with a $100 \%$ branching fraction, the other SUSY particles being decoupled. However, in the last part of this section dedicated searches for full spectrum SUSY models are detailed.

\subsection{SUSY - Strong production}

Strongly produced SUSY particles are expected to have the highest production cross-section of all SUSY processes, provided they are light enough to be produced at the LHC, and is one of the key process to possibly discover SUSY, see diagrams in Fig. 1 (a,b). Simplified models of gluino and squark pair production are considered : the gluino decays directly into two quarks and the lightest SUSY particle (LSP) with $100 \%$ branching fraction and the squark decays into a 


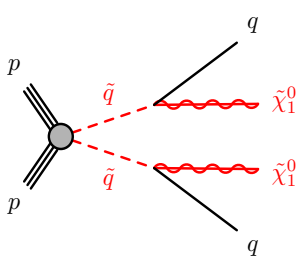

(a)

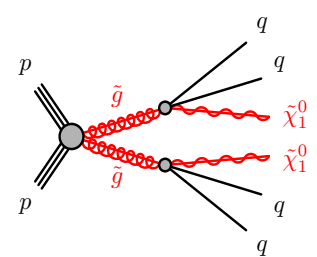

(b)

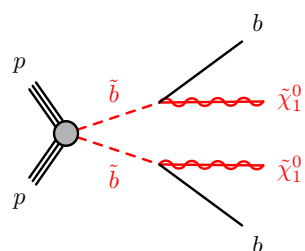

(c)

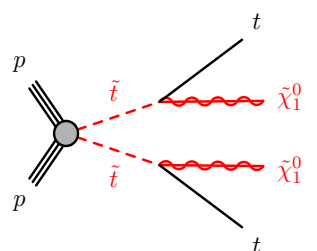

(d)

Figure 1: Diagrams for squark pair production (a), gluino pair production (b), for bottom squark pair production (c) and for top squark pair production (d), for simplified models reported in these proceedings.

quark and the LSP with $100 \%$ branching fraction. In both cases signal events are characterised by many jets (from 2 to 6), large MET and no lepton in the final state. The main SM backgrounds come from $\mathrm{Z}+\mathrm{jets}, \mathrm{W}+\mathrm{jets}$, top-antitop pair production and diboson processes. Figure 2 presents the expected exclusion limits and the discovery reaches, both for 300 and $3000 \mathrm{fb}^{-1}$. The Run 1 exclusion domain is also given. At the HL-LHC, for a low neutralino mass, the discovery reach and the exclusion limit are up to 3.1 and $3.5 \mathrm{TeV}$ respectively for the squark mass. The corresponding mass limits for the gluinos are up to 2.4 and $2.9 \mathrm{TeV}$ [9].
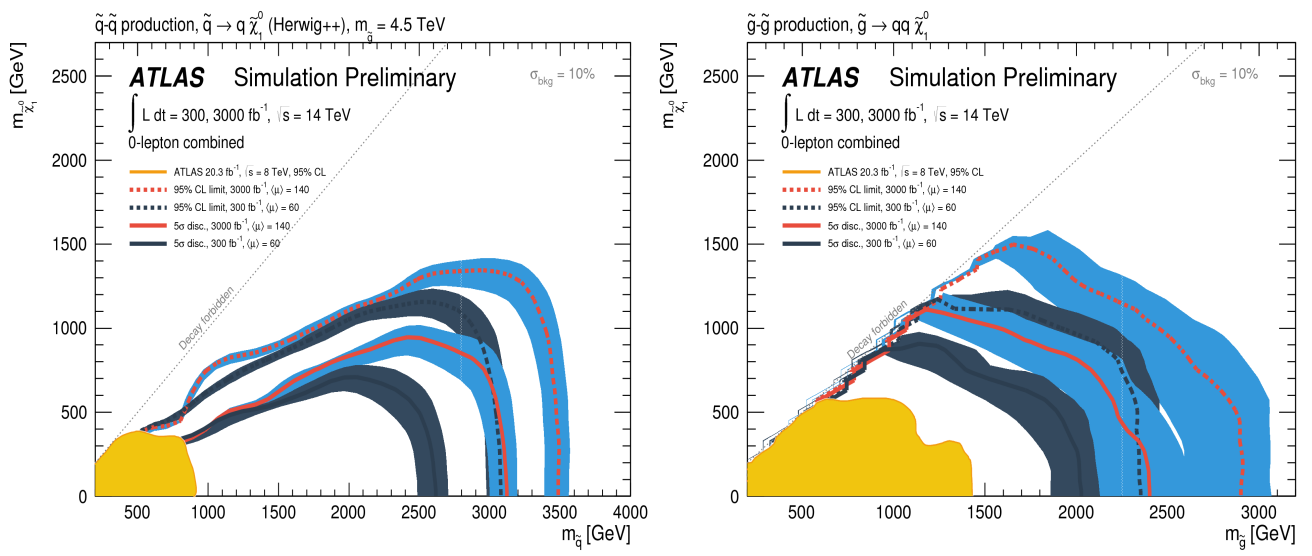

Figure 2: Expected 95\% CL exclusion contours (dashed) and $5 \sigma$ discovery contours (solid) for a luminosity of 300 (black) and 3000 (red) $\mathrm{fb}^{-1}$ for gluino and squark pair production. For squark pair production, the gluino mass is set to $4.5 \mathrm{TeV}$. The bands reflect the $1 \sigma$ uncertainty on the production cross section [9].

Search for the third generation squark were also studied [9-12], see diagrams in Fig. 1 (c,d). Search for bottom squark pair production are performed by selecting events with two b-taged jets and MET. At the HL-LHC, for a low neutralino mass, the discovery reach and the exclusion limit are up to 1.3 and $1.55 \mathrm{TeV}$, respectively, for the bottom squark mass. Events with 0 or 1 lepton, jets and MET are selected to search for top squark pair production. The discovery reach and the exclusion limit are up to 1.2 and $1.45 \mathrm{TeV}$, respectively, for the top squark mass.

\subsection{SUSY - Electroweak production}

If the squarks and gluinos are heavy, the direct production modes of charginos and neutralinos 

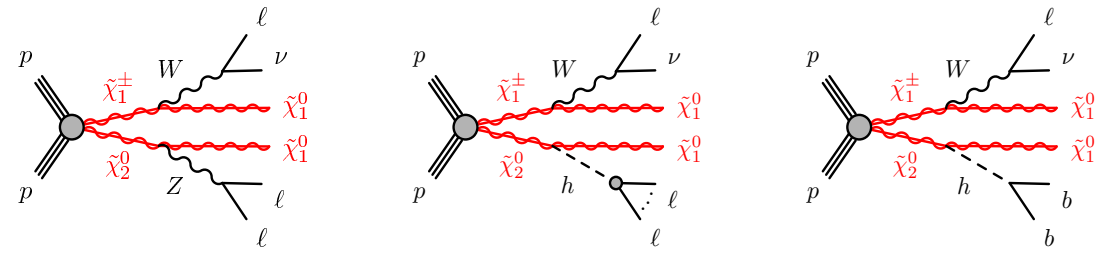

Figure 3: Diagrams for the direct production mode of a neutralino and a chargino in simplified models studied in these proceedings. The chargino (neutralino) is assumed to decay to a $\mathrm{W}(\mathrm{Z}$ or $\mathrm{H})$ boson and the LSP with $100 \%$ branching fraction.

are the best channels to discover SUSY. The electroweak SUSY processes are characterised by a smaller cross section production compared to the ones of squarks or gluinos. This channel will then highly benefit from the large increase of luminosity foreseen at the HL-LHC and a significantly enlarged discovery phase space is expected. Several final states have been studied by ATLAS and CMS; key channels are the 3 leptons + MET topologies, as illustrated in Fig. 3, the leptons coming from the $\mathrm{W}$ boson and the $\mathrm{Z}$ or $\mathrm{H}$ boson decays. The final state where the $\mathrm{H}$ boson decays into $\mathrm{a} \mathrm{b}$ quark pair is also considered. The results for the WZ and the WH mediated simplified models in the 3 lepton channel are presented in Fig. 4 [9]; the $\mathrm{H} \rightarrow \mathrm{b} \overline{\mathrm{b}}$ channel results are given in Fig. 5 (left) [8]. The effect on the limits of varying the contribution of the $\mathrm{WH}$ and $\mathrm{WZ}$ channels is reported in Fig. 5 (right) [11].
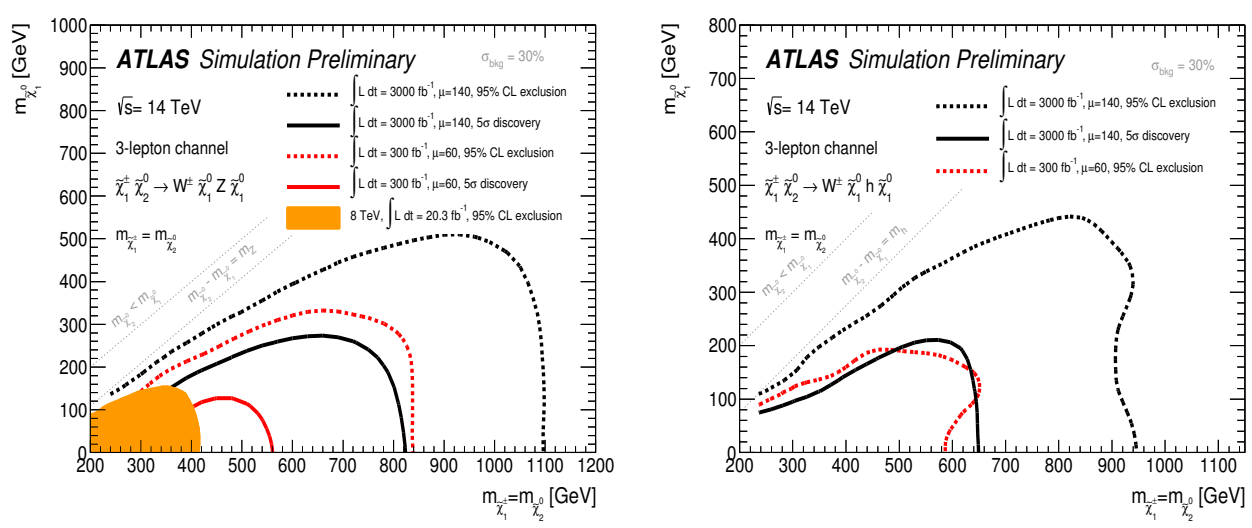

Figure 4: Expected 95\% CL exclusion contours (dashed) and $5 \sigma$ discovery contours (solid) for a luminosity of 300 (black) and 3000 (red) $\mathrm{fb}^{-1}$ in the LSP versus chargino mass plane for the WZ (left) and WH (right) mediated simplified model, for the 3-lepton channel [9].

A summary of the CMS projected discovery sensibilities in the case of simplified models is given in Fig. 6. The projection is based on the $8 \mathrm{TeV}$ data analysis [11,12]. At the HL-LHC, the $5 \sigma$ discovery is expected to be reached for masses up to $950 \mathrm{GeV}$ for charginos/neutralinos and up to $2.2 \mathrm{TeV}$ for gluinos.

\subsection{Full spectrum SUSY model}

A search for full spectrum SUSY models has been performed using multi-signature patterns, 

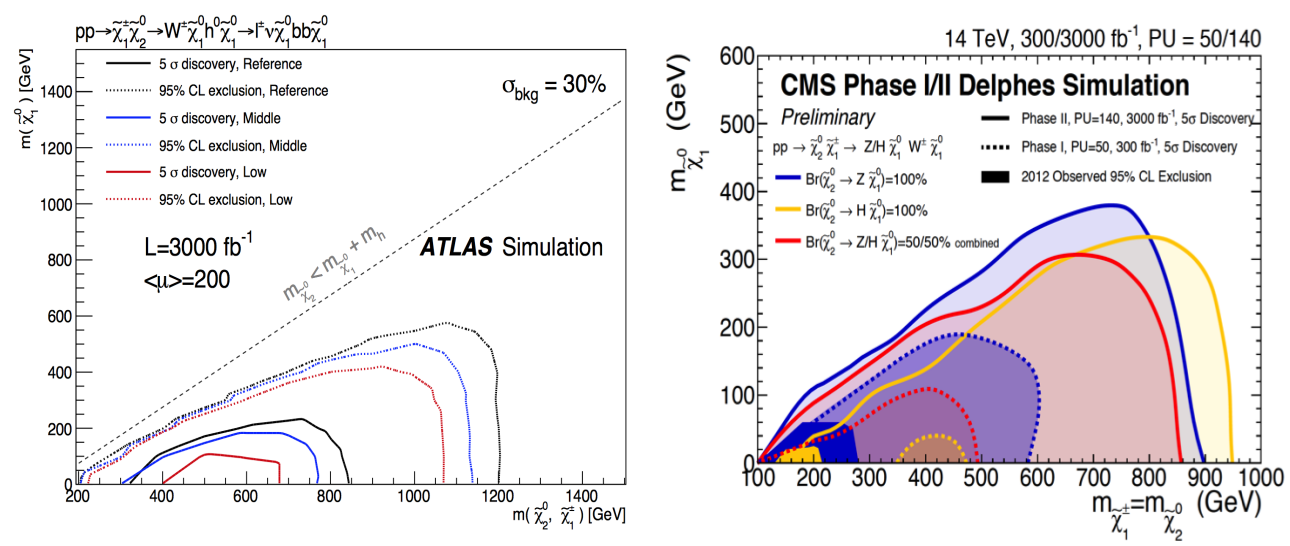

Figure 5: Left: expected 95\% CL exclusion contours (dashed black line) and $5 \sigma$ discovery contours (solid black line) for a luminosity of $3000 \mathrm{fb}^{-1}$ in the LSP versus chargino mass plane for the WH mediated simplified model, with $\mathrm{H} \rightarrow \mathrm{b} \bar{b}$ [8]. In this analysis the updated recommended value for the mean number of pileup $\langle\mu\rangle=200$ has been used. Right: expected $5 \sigma$ discovery contours in the same mass plane, for three different branching fraction assumptions for the next to lightest neutralino decays [11].

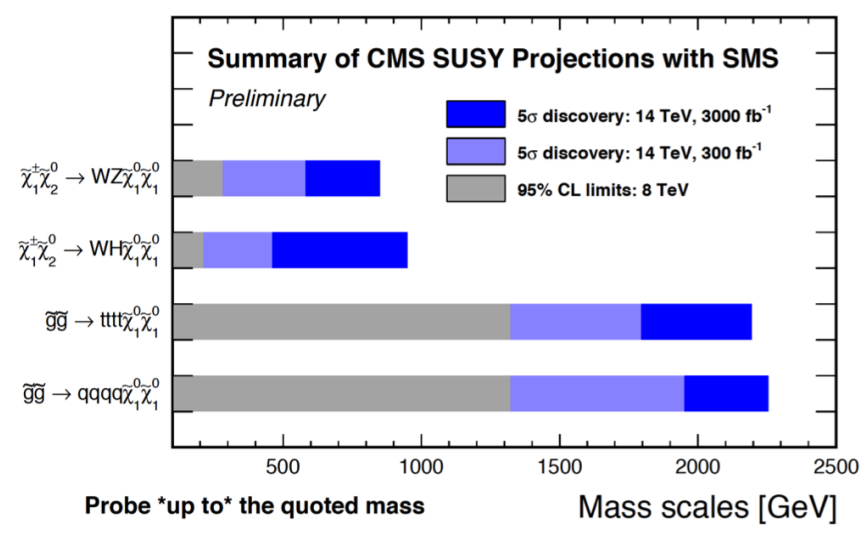

Figure 6: Projected performance of a selection of SUSY searches based on simplified models with an upgraded CMS detector at the LHC $\left(300 \mathrm{fb}^{-1}\right)$ and at the HL-LHC $\left(3000 \mathrm{fb}^{-1}\right)[11,12]$.

see Ref. [11]. It provides a powerful tool to identify the underlying SUSY spectrum of any potential signal. Five benchmark full-spectrum SUSY models are considered : the three first models are motivated by naturalness and differ by the mass of the sleptons and by the composition of neutralinos and charginos, the two other models contain coannihilation scenarios, which are motivated by their prediction of the relic density, leading to challenging experimental signature with compressed mass spectra. To study these scenarios, 9 experimental signatures have been investigated depending on the number of jets, b-taged jets and leptons in the final state, and using a variety of different kinematic variables. As an example, Fig. 7 presents the expected significance as a function of the integrated luminosity for the various benchmark models in the case of the search for direct top squark production in the single-lepton channel (left) and in the case of the multilepton 
channel search (right). A summary of the sensitivity in terms of number of sigmas of the 9 final states analysed for the 5 benchmark models considered is presented in Fig. 8.
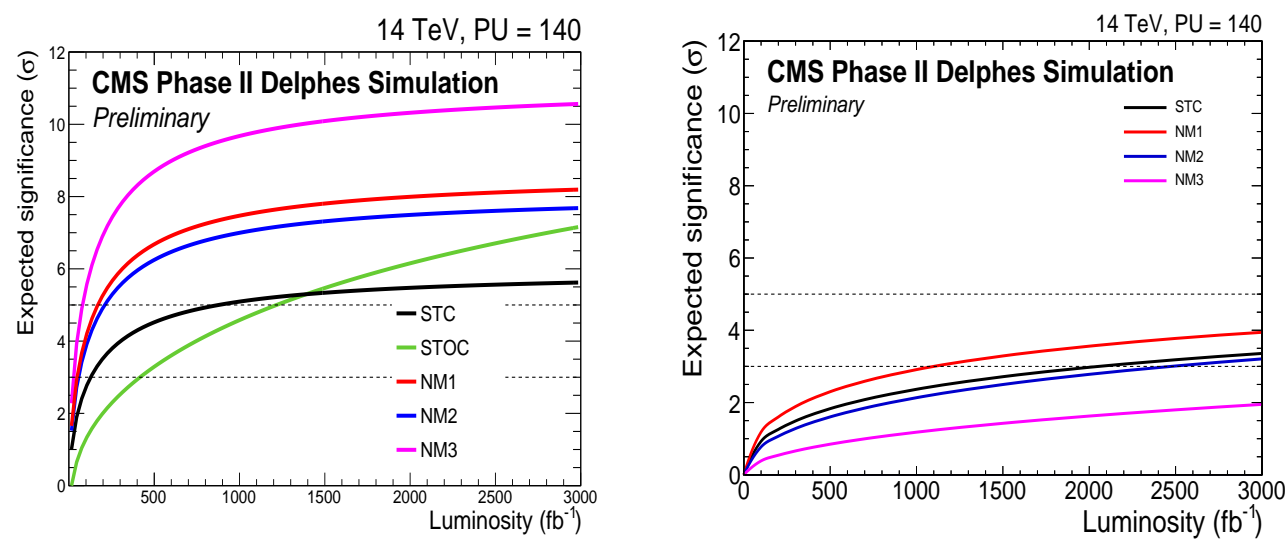

Figure 7: Expected significance for the various full-spectrum SUSY models, in the case of the single-lepton final state analysis (left) and the mulilepton final state analysis (right) [11].

\begin{tabular}{|c|c|c|c|c|c|c|}
\hline \multirow[t]{2}{*}{ Analysis } & \multirow{2}{*}{$\begin{array}{c}\text { Luminosity } \\
\left(\mathrm{fb}^{-1}\right)\end{array}$} & \multicolumn{5}{|c|}{ Model } \\
\hline & & NM1 & NM2 & NM3 & STC & STOC \\
\hline \multirow[t]{2}{*}{ all-hadronic $\left(H_{\mathrm{T}}-H_{\mathrm{T}}^{\text {miss }}\right)$ search } & 300 & & & & & \\
\hline & 3000 & & & & & \\
\hline \multirow[t]{2}{*}{ all-hadronic $\left(M_{\mathrm{T} 2}\right)$ search } & 300 & & & & & \\
\hline & 3000 & & & & & \\
\hline \multirow[t]{2}{*}{ all-hadronic $\widetilde{\mathrm{b}}_{1}$ search } & 300 & & & & & \\
\hline & 3000 & & & & & \\
\hline \multirow[t]{2}{*}{ 1-lepton $\widetilde{t}_{1}$ search } & 300 & & & & & \\
\hline & 3000 & & & & & \\
\hline \multirow[t]{2}{*}{ monojet $\widetilde{t}_{1}$ search } & 300 & & & & & \\
\hline & 3000 & & & & & \\
\hline \multirow[t]{2}{*}{$m_{\ell^{+} \ell^{-}}$kinematic edge } & 300 & & & & & \\
\hline & 3000 & & & & & \\
\hline \multirow[t]{2}{*}{ multilepton + b-tag search } & 300 & & & & & \\
\hline & 3000 & & & & & \\
\hline \multirow[t]{2}{*}{ multilepton search } & 300 & & & & & \\
\hline & 3000 & & & & & \\
\hline \multirow[t]{2}{*}{ ewkino WH search } & 300 & & & & & \\
\hline & 3000 & & & & & \\
\hline
\end{tabular}

$<3 \sigma|3-5 \sigma|>5 \sigma$

Figure 8: SUSY full spectrum search: performance in terms of sigmas of the 9 final state searches for the 5 benchmark models considered, for luminosities of 300 and $3000 \mathrm{fb}^{-1}$ [11].

\section{Prospects on BSM searches}

In addition to the SUSY prospects presented in the previous section, the ATLAS and CMS Collaborations have studied the physics potential for a series of beyond the standard model (BSM) processes. Projected performances for a selection of channels, taken from the CMS Technical Proposal (TP) for the HL-LHC phase 2 [5], are given in Fig. 9. The following searches are detailed in the rest of the section: searches for new heavy resonances in the dilepton, dijet and ditop final states, search for new physics in the multi b-quark final state, and search for dark matter candidates. 


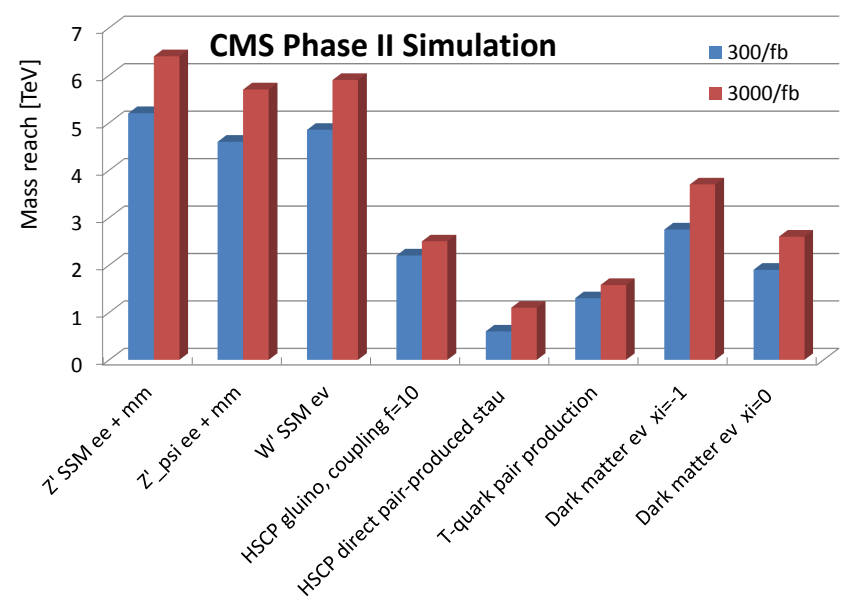

Figure 9: Projected performance of selected BSM searches with an upgraded CMS detector at the LHC (300 $\left.\mathrm{fb}^{-1}\right)$ and at the HL-LHC $\left(3000 \mathrm{fb}^{-1}\right)$. The $5 \sigma$ discovery reach in terms of particle mass is shown $[5,13]$.

\subsection{New heavy resonances in the dilepton decay channel}

Prospects of searches for heavy resonances have been studied by the ATLAS and CMS experiments [13-16]. Figure 10 presents the discovery potential for $\mathrm{Z}^{\prime} \rightarrow \mu \mu$ and $\mathrm{W}^{\prime} \rightarrow \ell v$ for various integrated luminosity values. For the commonly used benchmark of a Sequential Standard Model (SSM) Z', a heavy replica of the SM Z, the range of mass for discovery extends from 5.2 to $6.3 \mathrm{TeV}$ when the luminosity increases from 300 to $3000 \mathrm{fb}^{-1}$, in the case of the dimuon decay channel. In case of a discovery, the increase of luminosity is essential in order to characterise the signal and measure its properties, in particular from the angular distributions of the final state particles. The HL-LHC is needed to measure precisely the forward-backward asymmetry of any new spin-1 resonance $(\mathrm{M}=4 \mathrm{TeV})$, as in can be seen in Fig. 11 (left) [15]. The right plot in the figure presents the potential (separation in terms of number of sigmas) to distinguish between a Randall-Sundrum spin-2 graviton at $\mathrm{M}=4 \mathrm{TeV}$ and various alternative spins and/or production modes [16].

\subsection{New heavy resonances decaying in two scalar bosons}

A recent ATLAS result concerns the prospect at the HL-LHC of the search for a heavy resonance decaying into two scalar bosons, themselves decaying into a b-quark pair : $\mathrm{X} \rightarrow \mathrm{HH} \rightarrow$ $\mathrm{b} \bar{b} \mathrm{~b} \bar{b}$. The discovery of such a high mass resonance relies on the reconstruction performance of b-jets, and on the efficiency for reconstructing collimated ("boosted") objects originating from the decay of the resonance. The selection is optimised by the reconstruction of large radius jets [7]. A Kaluza-Klein graviton model is used as signal benchmark. The distribution of the invariant mass of the two large-radius jets is shown in Fig. 12 (left). The signal significance as a function of the resonance mass is given in Fig. 12 (right) : a significance of 4.4 is expected for a graviton mass of $2 \mathrm{TeV}$ for a luminosity of $3000 \mathrm{fb}^{-1}$. In this analysis the updated recommended value for the mean number of pileup $\langle\mu\rangle=200$ has been used. 

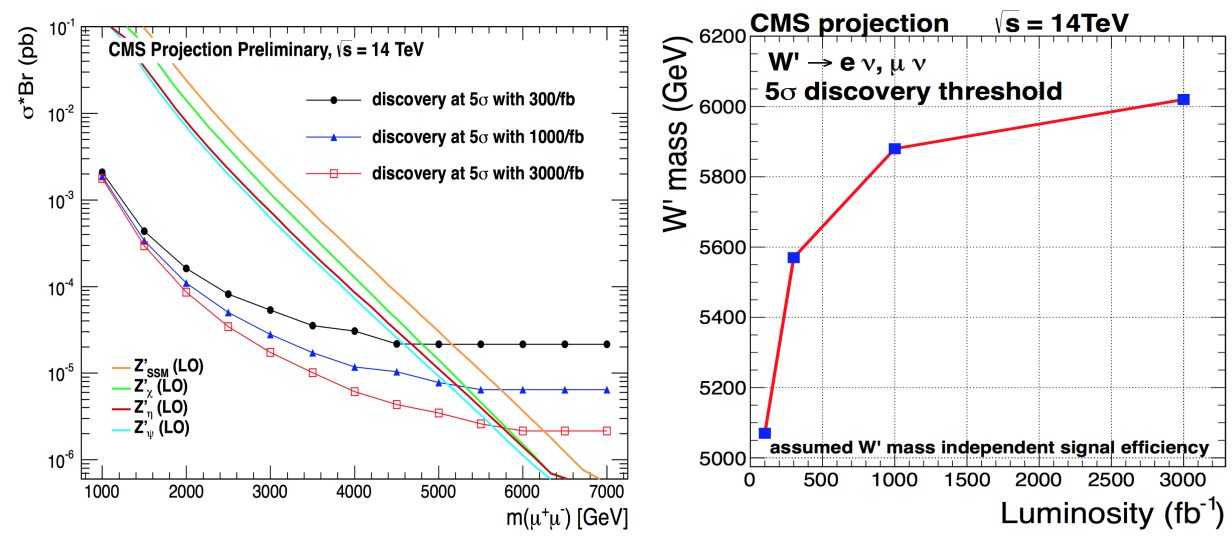

Figure 10: Projection of the $5 \sigma$ discovery reach for $14 \mathrm{TeV}$ for the SSM $Z^{\prime}$ in the dimuon decay channel (left) and the SSM W' in the $\mathrm{W}^{\prime} \rightarrow \ell v$ channel, for various luminosity scenarios [13].
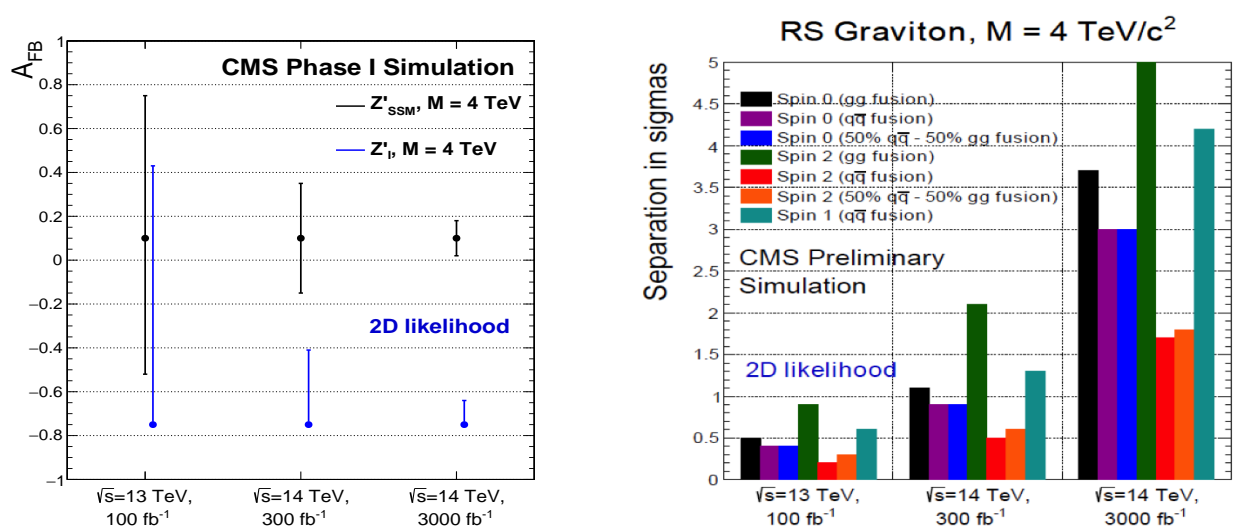

Figure 11: Left: expected measured value of the forward-backward asymmetry for two spin-1 $\mathrm{Z}^{\prime}$ models with $\mathrm{M}=4 \mathrm{TeV}$ and their $68 \%$ confidence level interval [15]. Right: expected potential (separation in sigmas) to distinguish between a Randall-Sundrum spin-2 graviton at $\mathrm{M}=4 \mathrm{TeV}$ and various alternative spins and/or production modes [16]. Both plots are given for various luminosity scenarios.

\subsection{Search for high mass resonances in dijet and ditop final states}

Search for dijet resonances have been investigated at the HL-LHC, using excited quark and quantum black hole production as signal benchmark models [17]. Smeared MC truth-jet information are used to estimated the sensitivity. Figure 13 (left) presents the expected signal cross section limits for various luminosity scenarios. The lower bound on the excited quark mass improves from 7.4 to $8.0 \mathrm{TeV}$ for luminosities from 300 to $3000 \mathrm{fb}^{-1}$. Prospects of the search for new massive neutral resonances decaying into a top-antitop quark pair are presented in Fig. 13 (right) in the lepton+jets channel. With 300 and $3000 \mathrm{fb}^{-1}$ of data, the (topcolor) $\mathrm{Z}^{\prime}$ model is expected to be excluded up to $Z^{\prime}$ masses of 3.3 and $5.5 \mathrm{TeV}$, respectively [14]. 

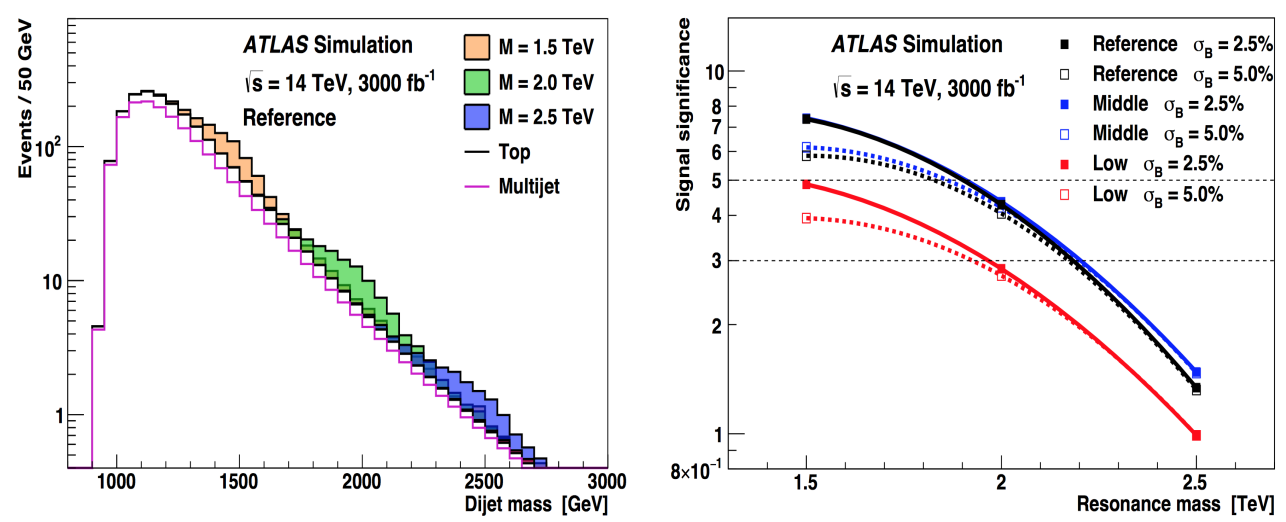

Figure 12: Left: expected di-jet mass distribution for events in the signal region for a luminosity of 3000 $\mathrm{fb}^{-1}$. Right: expected signal significance for a Kaluza-Klein graviton signal decaying into a $\mathrm{H}$ scalar boson pair, and $\mathrm{H} \rightarrow \mathrm{b} \overline{\mathrm{b}}$, with $3000 \mathrm{fb}^{-1}$, comparing the three detector upgrade scenarios (reference, low and middle). Two different assumptions on the systematic uncertainty in the background yield are tested: $2.5 \%$ and $5.0 \%$. In this analysis the mean number of pileup $\langle\mu\rangle=200$ has been used [7].
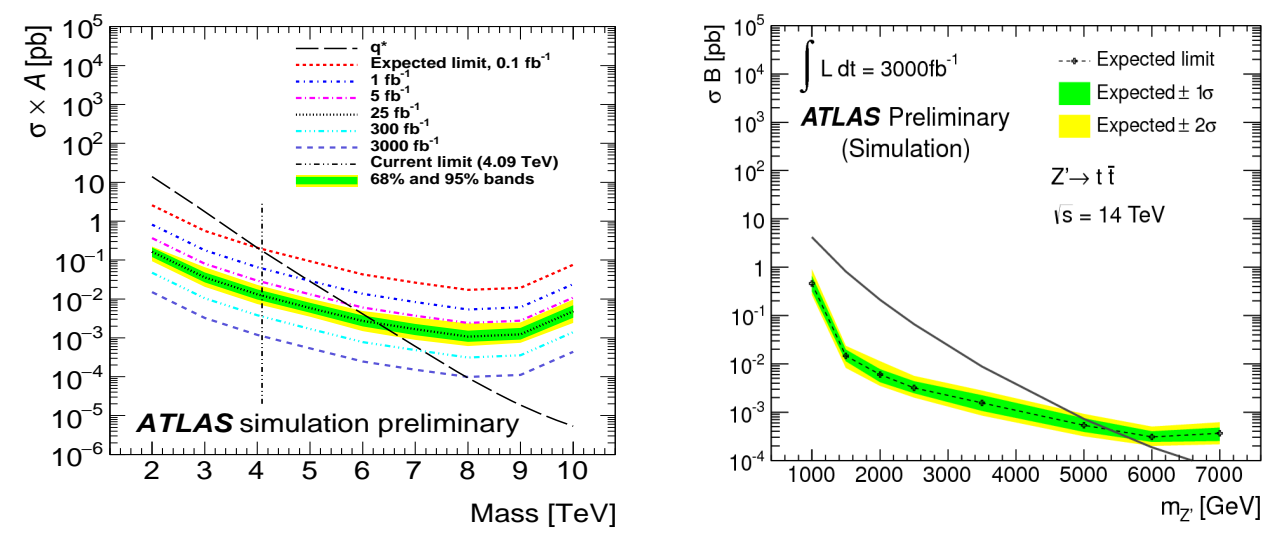

Figure 13: Left: expected limits on excited quark models for various luminosity scenarios, the dashed vertical line represents the current limit based on $20.3 \mathrm{fb}^{-1}$ of $8 \mathrm{TeV}$ data [17]. Right: expected limit for the $\mathrm{Z}^{\prime} \rightarrow \mathrm{t} \overline{\mathrm{t}}$ search in the lepton and MET channel for a luminosity of $3000 \mathrm{fb}^{-1}$ [14].

\subsection{Dark matter}

ATLAS and CMS have investigated the physics potential of the HL-LHC in the search for dark matter candidates $[15,18]$. ATLAS presented a study of the sensitivity of the mono-jet search to pair production of WIMP dark matter at the HL-LHC [18]. Events with a jet with transverse momentum $p_{T}$ above $300 \mathrm{GeV}$, a MET above $400 \mathrm{GeV}$, and passing a lepton veto are selected. Two assumptions on the systematic uncertainty in the background yield are tested: 1 and 5\%. Two different classes of models are investigated: the Effective Field Theory (EFT) and simplified models with an explicit light mediator. Figure 14 presents the discovery potential for dark matter signal with a D5 operator and for a dark matter mass $M_{D M}=50 \mathrm{GeV}$ for integrated luminosities of $300 \mathrm{fb}^{-1}$ and $3000 \mathrm{fb}^{-1}$. 
The factor 10 increase in the integrated luminosity leads to an improvement in the discovery reach for the suppression scale $\mathrm{M}$ from 2.2 to $2.6 \mathrm{TeV}$. Limitations of the EFT approach are discussed in the context of the energy reached at the LHC in Ref. [18].
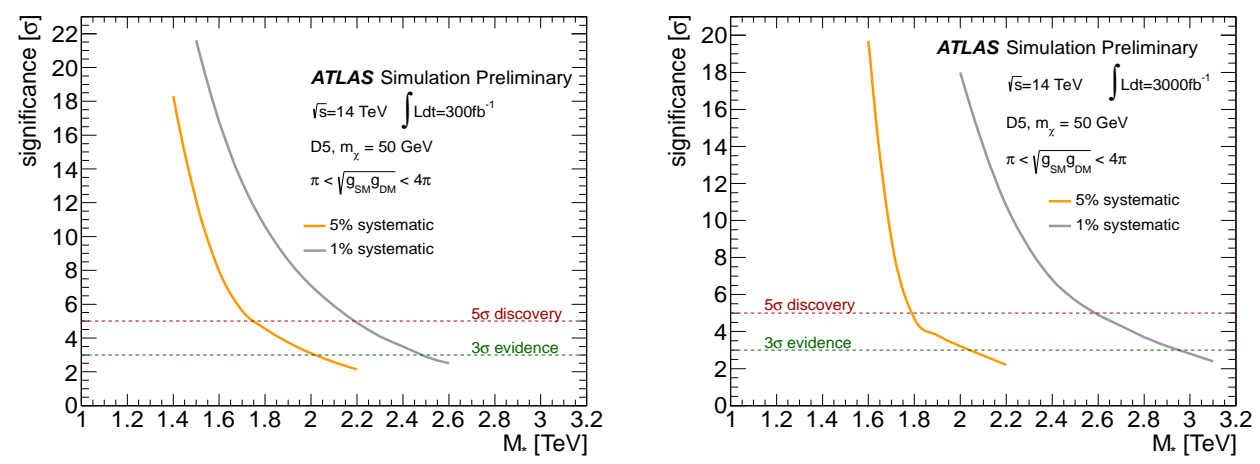

Figure 14: Discovery potential for dark matter signal with D5 operator and $M_{D M}=50 \mathrm{GeV}$ with $300 \mathrm{fb}^{-1}$ (left) and $3000 \mathrm{fb}^{-1}$ (right). These results assume that the EFT is a valid approach. Limitations of the EFT approach are discussed in the paper [18].

\section{Conclusions}

The observation of the new scalar boson $\mathrm{H}$ at a mass of $125 \mathrm{GeV}$ by the ATLAS and CMS experiments in July 2012 was a key discovery that opened an avenue of new questions: how is the electroweak scale stabilised? Is it accidental or natural? Is there new physics to be discovered at the TeV scale? The investigation of these issues is one of the major goals of the LHC for the next decade and beyond. The high-luminosity phase of the LHC (HL-LHC) is foreseen to start in 2026 and is expected to collect $3000 \mathrm{fb}^{-1}$ of pp collision data in about 10 years. These data will allow LHC physicists to make precise measurements of the standard model parameters in particular in the scalar sector, but also will considerably open the search for new physics proposed by many BSM models. In addition, the HL-LHC project is essential in order to identify any new physics hint that could possibly be observed during the LHC run 2 or run 3 or even after. Indeed a discovery could happen with various few-sigma excesses and interpretation of the new signal will require a large amount of data.

These proceedings have reviewed the prospects for the HL-LHC in terms of discovery potential (and exclusion limits) for SUSY processes as well as for a selection of BSM models. The performances for two integrated luminosity scenarios have been estimated and compared: for 300 and $3000 \mathrm{fb}^{-1}$. At the HL-LHC, much gain is expected for weakly produced SUSY particles because they are characterised by small production cross sections. If gluinos and squarks are heavy, search for electroweakinos will be the best channel to discover SUSY. At the HL-LHC, charginos/neutralinos are expected to be discovered $(5 \sigma)$ up to masses of about $950 \mathrm{GeV}$. In the case of strong production, the mass reach for gluinos goes up to $2.2 \mathrm{TeV}$. A SUSY full spectrum analysis using multi-signature patterns has also been detailed and provides a powerful tool to potentially identify the underlying SUSY spectrum. Prospects for several BSM searches have been 
presented: searches for new heavy resonances in various decay channels and search for dark matter candidates.

\section{References}

[1] ATLAS Collaboration, “The ATLAS Experiment at the CERN Large Hadron Collider", JINST 03 (2008) S08003

[2] CMS Collaboration, “The CMS experiment at the CERN LHC”, JINST 03 (2008) S08004

[3] ATLAS Collaboration, Physics Studies for ATLAS Upgrades (web page): https://twiki.cern.ch/twiki/bin/view/AtlasPublic/UpgradePhysicsStudies

[4] CMS Collaboration, CMS Upgrade and physics documents (web page): https://twiki.cern.ch/twiki/bin/view/CMSPublic/PhysicsResultsFP

[5] CMS Collaboration, "Technical Proposal for the Phase-II Upgrade of the CMS Detector", CMS-TDR-15-02, CERN-LHCC-2015-010

[6] CMS Collaboration, “CMS Phase II Upgrade Scope Document”, CERN-LHCC-2015-019

[7] ATLAS Collaboration, “ATLAS phase-II Upgrade Scoping Document”, CERN-LHCC-2015-020

[8] ATLAS Collaboration, "Prospect for a search for direct pair production of a chargino and a neutralino decaying via a W boson and the lightest Higgs boson in final states with one lepton, two b-jets and missing transverse momentum at the high luminosity LHC with the ATLAS Detector", ATL-PHYS-PUB-2015-032

[9] ATLAS Collaboration, "Search for Supersymmetry at the high luminosity LHC with the ATLAS experiment”, ATL-PHYS-PUB-2014-010

[10] ATLAS Collaboration, "Prospects for benchmark Supersymmetry searches at the high luminosity LHC with the ATLAS Detector", ATL-PHYS-PUB-2013-011

[11] CMS Collaboration, "Supersymmetry discovery potential in future LHC and HL-LHC running with the CMS detector", CMS-PAS-SUS-14-012

[12] CMS Collaboration, "Study of the Discovery Reach in Searches for Supersymmetry at CMS with $3000 \mathrm{fb}^{-1}$, CMS-PAS-FTR-13-014

[13] CMS Collaboration, "Projected Performance of an Upgraded CMS Detector at the LHC and HL-LHC: Contribution to the Snowmass Process", CMS-NOTE-2013-002, arXiv:1307.7135

[14] ATLAS Collaboration, "Studies of Sensitivity to New Dilepton and Ditop Resonances with an Upgraded ATLAS Detector at a High-Luminosity LHC”, ATL-PHYS-PUB-2013-003

[15] CMS Collaboration, "The potential to study exotic physics signatures at HL-LHC using the phase 2 upgraded CMS detector", CMS-PAS-EXO-14-007

[16] CMS Collaboration, Physics Performance for 2nd ECFA workshop, https://twiki.cern.ch/twiki/pub/CMSPublic/PhysicsResultsFP/ECFA-CMSPublicResults.pdf

[17] ATLAS Collaboration, "Dijet resonance searches with the ATLAS detector at 14 TeV LHC", ATL-PHYS-PUB-2015-004

[18] ATLAS Collaboration, "Sensitivity to WIMP Dark Matter in the Final States Containing Jets and Missing Transverse Momentum with the ATLAS Detector at $14 \mathrm{TeV}$ LHC", ATL-PHYS-PUB-2014-007 